\title{
Unsteady Heat Transfer from an Equilateral Triangular Cylinder in the Unconfined Flow Regime
}

\author{
Amit Dhiman and Radhe Shyam \\ Department of Chemical Engineering, Indian Institute of Technology Roorkee, Uttarakhand 247 667, India \\ Correspondence should be addressed to Amit Dhiman, dhimuamit@rediffmail.com
}

Received 14 January 2011; Accepted 14 February 2011

Academic Editors: H. Hadavinia, F. H. Moukalled, and S. C. M. Yu

Copyright ( $) 2011$ A. Dhiman and R. Shyam. This is an open access article distributed under the Creative Commons Attribution License, which permits unrestricted use, distribution, and reproduction in any medium, provided the original work is properly cited.

\begin{abstract}
Effects of Reynolds number on the heat transfer characteristics of a long (heated) equilateral triangular cylinder are investigated for the range of conditions $\mathrm{Re}=50-150$ (in the steps of 10) and Prandtl number $=0.71$ (air) in the unconfined unsteady crossflow regime. In order to simulate the present situation, the computational grid is created by using commercial grid generator GAMBIT and the numerical computations are carried out by using FLUENT (6.3). The SIMPLE method is used to solve continuity, Navier-Stokes and energy equations along with the appropriate boundary conditions. The second order upwind scheme is used to discretize the convective terms, while the central difference scheme is used to discretize the diffusive terms in the governing equations. The present results are in an excellent agreement with the literature values. The temperature isotherms and temporal history of Nusselt number are presented in detail. The local as well as time-averaged Nusselt numbers are calculated. The timeaveraged Nusselt number increases with increasing Reynolds number for the fixed value of the Prandtl number. Finally, the present numerical results are used to develop the simple heat transfer correlation for the range of conditions covered here.
\end{abstract}

\section{Introduction}

The fluid flow over bluff bodies (e.g., cylinders) has been the topic of intense research for a century or so because of its importance in various engineering applications, such as electronic cooling, heat exchange systems, cooling towers, design of vortex flow meters and flow dividers, probes and sensors, and so forth. On the other hand, in spite of such wide applications, limited information is available in the open literature as far as forced convection flow and/or heat transfer from noncircular obstacles such as triangular, trapezoidal, and rectangular cylinders are concerned [1-17]. This has motivated us to examine the heat transfer around an obstacle of triangular cross-section in the unsteady flow regime. For instance, as far as known to us, the fluid flow and/or heat transfer across a triangular cylinder are investigated only by a few researchers in both unbounded [6-10] and bounded [11-13] configurations. The numerical investigations dealing with the heat transfer from a long unconfined square cylinder can be found in [18-22]. Further, a healthy literature on the heat transfer from the unconfined obstacle of circular cross-section can be found elsewhere
[23-35]. This study is concerned with the heat transfer from a long equilateral triangular obstacle in the unsteady unconfined flow regime.

Jackson [6] examined the onset of periodic behavior over variously shaped bodies, such as circular and elliptical cylinders, triangular prisms, and flat plates, by using finite element method in the two-dimensional unbounded flow regime. Zielinska and Wesfreid [7] performed 2-D numerical simulation of the flow through a triangular cylinder to obtain global modes of wake flow for the range of conditions: Reynolds number $=34-50$ and blockage ratios $=1 / 5$ and $1 / 15$ in the bounded configuration. Wesfreid et al. [8] carried out 2-D numerical simulations for the fluid flow over an equilateral triangular bluff body for a blockage ratio of $1 / 15$. They reported the critical Reynolds number of 36.2 for the onset of the oscillations. De and Dalal [9] carried out 2-D numerical investigations of the fluid flow across an equilateral triangular cylinder in the unconfined space in the Reynolds number range $10 \leq \mathrm{Re} \leq 250$. They studied global modes in wake flow, and the value of critical Reynolds number for triangular cylinder is reported to be 39.9. 
As far as heat transfer from a long triangular obstacle is concerned, Chattopadhyay [11] has done a 2-D numerical study on the augmentation of heat transfer in a channel due to triangular prism at very high Reynolds numbers in the order of 10,000. Abbassi et al. [12] carried out the 2-D forced convection in a channel with a built-in triangular prism for the Reynolds number $=20-250$ and Prandtl number of 0.71 for a fixed blockage ratio of $1 / 4$. They found an enhancement of about $85 \%$ in the heat transfer at a Reynolds number of 250. Recently, De and Dalal [13] investigated the flow and heat transfer across an equilateral triangular cylinder placed in a 2 -D confined space with blockage ratios of $1 / 12 \leq \beta \leq 1 / 3$ for the range $80 \leq \mathrm{Re} \leq 200$ and $\operatorname{Pr}=0.71$. They reported the variation of drag coefficient, Strouhal number, lift coefficient, and Nusselt number with respect to Reynolds number. More recently, Dalal et al. [10] proposed a novel finite-volume formulation for unsteady solutions on complex geometries. They presented a computational study of 2-D laminar flow and heat transfer past a triangular cylinder in free stream for the range $10 \leq \operatorname{Re} \leq 200$ and $\operatorname{Pr}=0.71$. The time-averaged Nusselt number increases quasilinearly with increasing value of the Reynolds number. However, very limited information is available on the variation of the local Nusselt number, and no information is available on the temporal variation of the Nusselt number with Reynolds number.

Thus, as far as known to us, it can be summarized that there is only one limited study due to Dalal et al. [10]. The work in [10] is available in the open literature on the heat transfer from an unconfined equilateral triangular cylinder in the 2-D unsteady flow regime. Therefore, the aim of this work is to provide the detailed systematic study on the heat transfer from a cylinder of triangular cross-section in the unconfined (periodic) flow regime. In particular, the heat transfer from a long equilateral triangular cylinder is investigated for the Reynolds number range $\mathrm{Re}=50-150$ (in the steps of 10) for air $(\mathrm{Pr}=0.71)$. The heat transfer characteristics around the triangular obstacle are presented by instantaneous isotherm profiles and by temporal history of the Nusselt number at various values of the Reynolds number for the fixed value of the Prandtl number. The variation of the local Nusselt number on the surfaces of the triangular cylinder is shown. Finally, the simple heat transfer correlation is obtained in order to calculate the time-averaged Nusselt number for the range of conditions covered here.

\section{Problem Statement and Mathematical Formulation}

In the present study, the two-dimensional and unsteady (periodic) flow of an incompressible fluid (with a uniform velocity, $U_{\infty}$, and uniform temperature, $T_{\infty}$, at the inlet) across a long equilateral triangular cylinder (of side $d$ ) is considered, as shown in Figure 1. The surface of the triangular obstacle is maintained at a constant temperature, $T_{w}^{*}\left(>T_{\infty}\right)$. The thermophysical properties of the streaming fluid are assumed to be independent of the temperature, and the viscous dissipation effects are also neglected. Here, the unconfined flow condition is simulated by creating an artificial long channel; however, as the computational domain

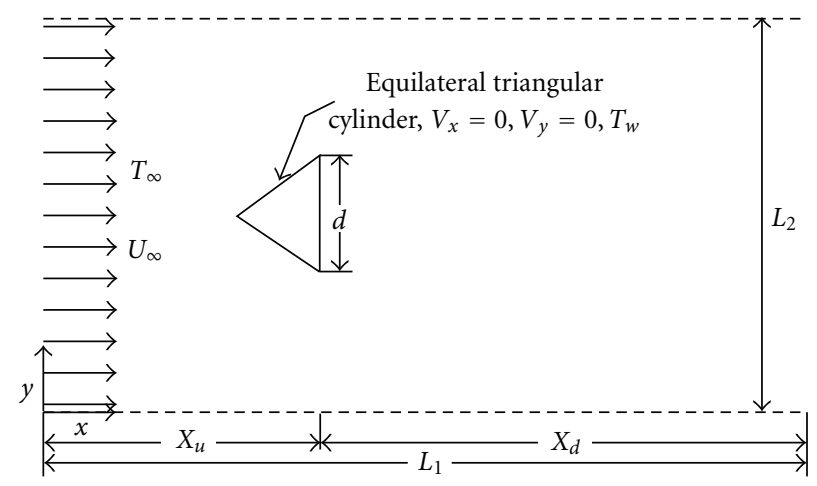

FIGURE 1: Schematics of the unconfined flow across an equilateral triangular cylinder.

has to be finite, the nondimensional upstream distance, $X_{u} / d$, is taken as 12 , and the nondimensional downstream distance between the rear surface of the triangular cylinder and the exit plane $\left(X_{d} / d\right)$ is taken as 20 , with the total nondimensional length of the computational domain $\left(L_{1} / d\right)$ of 32 in the axial direction. The height of the computational domain $\left(L_{2} / d\right)$ is used as 30 in the lateral direction.

The dimensionless equations of the continuity, $x$-, and $y$-momentums, and energy balances are given by (1)-(4). Here, the Reynolds and Prandtl numbers are defined as $\mathrm{Re}=$ $d U_{\infty} \rho / \mu$ and $\operatorname{Pr}=\mu c_{p} / k$, respectively.

\section{Continuity Equation:}

$$
\frac{\partial V_{x}}{\partial x}+\frac{\partial V_{y}}{\partial y}=0
$$

x-Momentum Equation:

$$
\frac{\partial V_{x}}{\partial t}+\frac{\partial\left(V_{x} V_{x}\right)}{\partial x}+\frac{\partial\left(V_{y} V_{x}\right)}{\partial y}=-\frac{\partial p}{\partial x}+\frac{1}{\operatorname{Re}}\left(\frac{\partial^{2} V_{x}}{\partial x^{2}}+\frac{\partial^{2} V_{x}}{\partial y^{2}}\right)
$$

y-Momentum Equation:

$$
\frac{\partial V_{y}}{\partial t}+\frac{\partial\left(V_{x} V_{y}\right)}{\partial x}+\frac{\partial\left(V_{y} V_{y}\right)}{\partial y}=-\frac{\partial p}{\partial y}+\frac{1}{\operatorname{Re}}\left(\frac{\partial^{2} V_{y}}{\partial x^{2}}+\frac{\partial^{2} V_{y}}{\partial y^{2}}\right) .
$$

Energy Equation:

$$
\frac{\partial \theta}{\partial t}+\frac{\partial\left(V_{x} \theta\right)}{\partial x}+\frac{\partial\left(V_{y} \theta\right)}{\partial y}=\frac{1}{\operatorname{RePr}}\left(\frac{\partial^{2} \theta}{\partial x^{2}}+\frac{\partial^{2} \theta}{\partial y^{2}}\right)
$$

The following boundary conditions in their dimensionless forms are used (Figure 1).

(i) At the inlet boundary: isothermal fluid is flowing uniformly from left to right, that is, $V_{x}=1, V_{y}=0$, and $\theta=0$. 
(ii) On the surface of the triangular obstacle: No-slip condition is used, that is, $V_{x}=0, V_{y}=0$ and $\theta=1$.

(iii) At the exit boundary: the default outflow boundary condition in FLUENT, which assumes a zero diffusion flux for all flow variables, is used. This is similar to Neumann boundary condition as $\partial V_{x} / \partial x=$ $0, \partial V_{y} / \partial x=0$, and $\partial \theta / \partial x=0$.

(iv) On the top and bottom boundaries: the symmetric boundary condition used by FLUENT is used.

The above continuity, $x$ - and $y$-momentum, and thermal energy equations (1)-(4) along with the above-noted boundary conditions are solved for the fluid flow and heat transfer over a long equilateral triangular obstacle to obtain velocity, pressure, and temperature fields. However, the present study is concerned with the heat transfer from an equilateral triangular cylinder in the unconfined unsteady flow regime.

\section{Numerical Details}

In the present work, the grid structure consists of nonuniform orthogonal cells having 116500 cells with 200 grid points on each side of the triangular cylinder and a very fine grid of cell size, $\delta=0.0015 d$, is used near the triangular cylinder, and larger size grids are used away from the triangular cylinder (details are given in Section 3.1), as shown in Figure 2. The number of grid points and their distribution are an important matter in such unsteady laminar flow over cylinders, because of the complex phenomena existing in this type of flow due to separation and vortex shedding, and so forth.

The fluid flow and heat transfer over a long unconfined equilateral triangular obstacle are solved by using commercial CFD solver FLUENT (6.3). FLUENT uses finite volume method, according to which, it is assumed that volume is made up of a large number of small control volumes, which are regular parallelepiped. The governing equations are valid over all such control volumes. Hence, equations can be discretized to be written in algebraic form to be solved. The values at the finite volumes can be summed up to get the value over entire domain. The grid is generated by using GAMBIT. The second-order upwind scheme is used to discretize convective terms while the diffusive terms are discretized by central difference scheme. The resulting algebraic equations are solved by Gauss-Siedel iterative scheme. A convergence criterion of $10^{-20}$ is found to be adequate for continuity, $x$ - and $y$-components of NavierStokes, and energy equations to determine the flow and heat transfer parameters here. Also, the solution is assumed to have converged, when it shows more than 10 constant periodic cycles in the time history profiles.

3.1. Choice of Numerical Parameters. In this subsection, heat transfer calculations are carried out in order to fix the grid size, upstream and downstream distances, and the height of the computational domain for the heat transfer parameters in the unconfined unsteady regime.

For the grid resolution study, several nonuniform orthogonal grids (with 100 and 200 grid points on each side

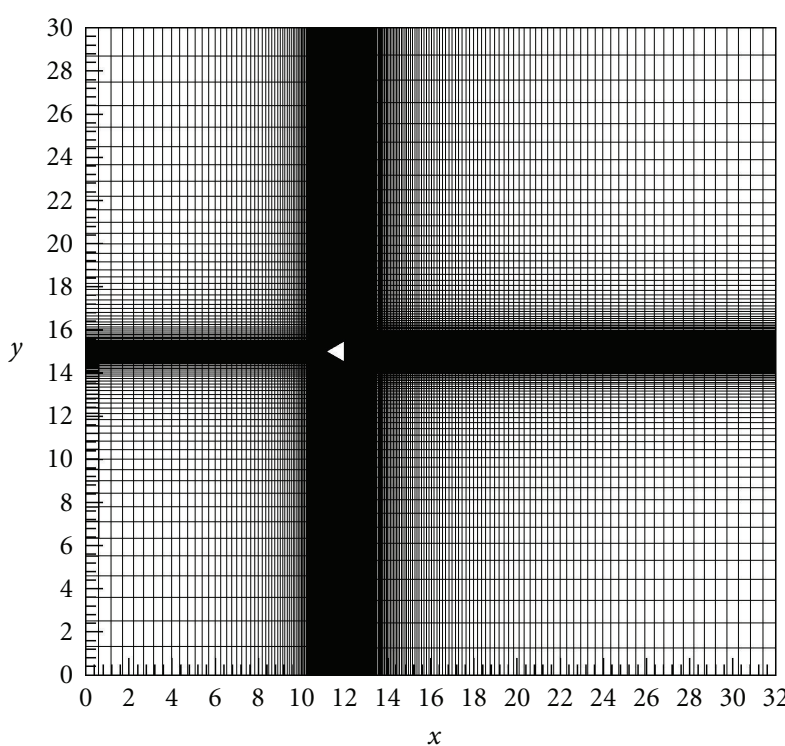

FIGURE 2: Nonuniform computational grid structure.

of the triangular obstacle and having a first grid point at distances of $0.004 d, 0.0015 d$, and $0.001 d$ ) are examined for $X_{u}=12 d, X_{d}=20 d$, and $L_{2}=30 d$ (Table 1). The computational grid structure used in this work is presented in Figure 2. Table 1 also presents the grid independence results for the value of the Reynolds number of 100 and Prandtl number of 0.71 . The percentage relative differences in the values of the time-averaged Nusselt number are found to be less than $0.4 \%$ for the grid with 200 grid points on the each side of the triangular cylinder and having a first grid point at a distance of $0.004 d$ with respect to the finest grid size $(\delta=0.001 d)$ for the Reynolds number of 100 and Prandtl number of 0.71 . However, the percentage relative differences in the values of the time-averaged Nusselt number are found to be only less than $0.2 \%$ for the grid with 200 grid points on the each side of the triangular cylinder and having a first grid point at a distance of $0.0015 d$ with respect to the finest grid size of $0.001 \mathrm{~d}$. Thus, the grid with 200 grid points on the surface of the triangular cylinder and $\delta=0.0015 d$ are used in all the computations reported in this work.

The domain independence study is carried out for two values of the height of the computational domain, that is, $L_{2}=20 d$ and $30 d$ for $\delta=0.0015 d$ with 200 grid points prescribed on each surface of the triangular cylinder. The percentage differences in the values of time-averaged Nusselt number are found to be less than $0.3 \%$ for the value of Reynolds number of 100 and Prandtl number of 0.71 . Therefore, in this work, the computational domain height of $30 d$ is used with $\delta=0.0015 d$ and 200 grid points on each surface of the triangular cylinder for $X_{u}=12 d$ and $X_{d}=20 d$.

In order to determine the effect of the upstream distance on the heat transfer parameters, two values of the upstream distance are used, that is, $X_{u}=9 d$ and $12 d$ with $\delta=0.0015 d$ and 200 grid points on the surface of the cylinder for $X_{d}=$ $20 d$, for $\operatorname{Re}=100$, and $\operatorname{Pr}=0.71$. The percentage differences 
TABLE 1: Grid independence study for the value of the Reynolds number of 100 and Prandtl number of 0.71 .

\begin{tabular}{llll}
\hline Re & $\begin{array}{l}\text { Total number of } \\
\text { cells in the domain }\end{array}$ & $\begin{array}{l}\text { Spacing of the first } \\
\text { grid point from the } \\
\text { cylinder }\end{array}$ & $\begin{array}{l}\text { Time-averaged } \\
\text { Nusselt number }\end{array}$ \\
\hline \multirow{3}{*}{100} & 116500 & $0.004 d$ & 5.5961 \\
& 116500 & $0.0015 d$ & 5.5843 \\
116500 & $0.001 d$ & 5.5741 \\
\hline
\end{tabular}

TABLE 2: Validation of present flow results with literature values for $\operatorname{Re}=50,100$ and 150 .

\begin{tabular}{lcccc}
\hline \multirow{2}{*}{ Re } & \multicolumn{2}{c}{ Present work } & \multicolumn{2}{c}{ De and Dalal [9] } \\
& St & $\overline{C_{D}}$ & St & $\overline{C_{D}}$ \\
\hline 50 & 0.1455 & 1.5257 & 0.1505 & 1.5420 \\
100 & 0.1916 & 1.7316 & 0.1966 & 1.7607 \\
150 & 0.2041 & 1.8937 & 0.2015 & 1.8750 \\
\hline
\end{tabular}

TABLE 3: Validation of time-averaged Nusselt number with the results of Dalal et al. [10] for $\operatorname{Re}=80,100$, and 150 for $\operatorname{Pr}=0.71$.

\begin{tabular}{lccc}
\hline Source & $\mathrm{Re}=80$ & $\mathrm{Re}=100$ & $\mathrm{Re}=150$ \\
\hline Present work & 4.8711 & 5.5843 & 7.0447 \\
Dalal et al. [10] & 4.954 & 5.67 & 7.31 \\
\hline
\end{tabular}

in the values of time-averaged Nusselt number are found to be less than $0.25 \%$. Thus, the upstream distance of $12 d$ is used here.

In summary, the following parameters have been used for the generation of results presented here as $X_{d}=20 d$, $X_{u}=12 d, L_{2}=30 d$ for the grid size of 116500 with 200 grid points on the each surface of the triangular cylinder, and $\delta=0.0015 d$.

\section{Results and Discussion}

In this study, the two-dimensional numerical simulations are carried out in the full computational domain for the range of conditions: Reynolds number $=50-150$ (in the steps of 10) and Prandtl number $=0.71$ (air).

4.1. Validation of Results. The comparison of present flow results and that of De and Dalal [9] of time-averaged drag coefficient and Strouhal number for the Reynolds number of 50,100, and 150 are shown in Table 2. An excellent agreement between the two studies can be seen in this table. For instance, the percentage deviations in the values of the time-averaged drag coefficient are found to be less than 3.5\%, less than $2.6 \%$, and less than $1.3 \%$ for the values of the Reynolds number of 50,100, and 150, respectively. However, the corresponding deviations in the values of the Strouhal number are found to be less than $1.1 \%$, less than $1.7 \%$, and less than $1.0 \%$ for the values of the Reynolds number of 50 , 100 , and 150 , respectively.
For the validity of the heat transfer results, the present heat transfer results are compared with Dalal et al. [10] in Table 3. Again, the excellent agreement can be seen here between the two studies. The percentage deviations in the values of the time-averaged Nusselt number are found to be less than $1.7 \%$, less than $1.55 \%$, and less than $3.65 \%$ for the values of the Reynolds number of 80,100 , and 150, respectively. This validates the present numerical solution procedure.

The following subsections present the details about the temperature fields around the triangular obstacle, variation of the Nusselt number with time (i.e., temporal history of the Nusselt number), the variation of local and time-averaged Nusselt number with Reynolds number, and so forth.

4.2. Isotherm Patterns. The effects of Reynolds number on the heat transfer around the long equilateral triangular obstacle are presented via instantaneous isotherm profiles for the fixed value of the Prandtl number of 0.71. Figures 3$5(\mathrm{a}-\mathrm{d})$ present the representative instantaneous temperature profiles around the triangular cylinder for the Reynolds numbers of 50,100, and 150 for the four successive moments of time, which span over the whole period (i.e., $t=0$, $T / 4,2 T / 4$, and $3 T / 4$, for instance). Note that in Figures 3, 4, and 5, (a) will be repeated after (d) for the next cycle. However, the magnified views of isotherms in the vicinity of the triangular cylinder can be seen in Figures 3-5(e-h). It is clear from these figures that a temperature street is formed behind the triangular obstacle, which is very similar to Karman vortex street for all the values of the Reynolds number covered in this work. It can also be seen in these figures that the top and bottom surfaces of the triangular obstacle have the maximum clustering of isotherms, which result in high temperature gradients as compared to the rear surface of the triangular cylinder. Also, as the Reynolds number increases, the distribution of isotherms on the rear surface of the triangular cylinder affects more than that of the top and bottom surfaces of the triangular cylinder with time due to vortex shedding for the range of conditions covered here (Figures 3, 4, and 5).

4.3. Local Nusselt Number. In this study, the local Nusselt number $(\mathrm{Nu})$ is defined as $-\partial \theta / \partial n$, where $n$ is the cylinder surface normal direction. Due to the singularity at the corner of the triangular obstacle, special attention is paid to the role of the grid size on the value of the local Nusselt number at each corner of the long triangular cylinder. It is found that the grid with 200 cells on each side of the triangular obstacle is sufficiently fine to obtain results that are essentially grid independent. The variation of the instantaneous local Nusselt number for the four successive moments of time, which span over the whole period, for Reynolds numbers of 50, 100, and 150 is shown in Figures $6(\mathrm{a}-\mathrm{c})$. The corresponding enlarged views are presented in Figures $6(\mathrm{~d}-\mathrm{f})$. The local Nusselt number at each corner of the triangular cylinder increases with increasing value of the Reynolds number. It can be seen from these figures that there exists a local minimum in the value of the local 


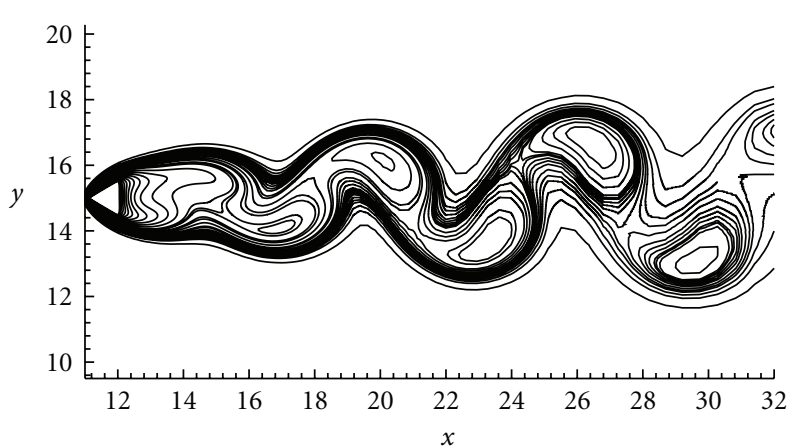

(a)

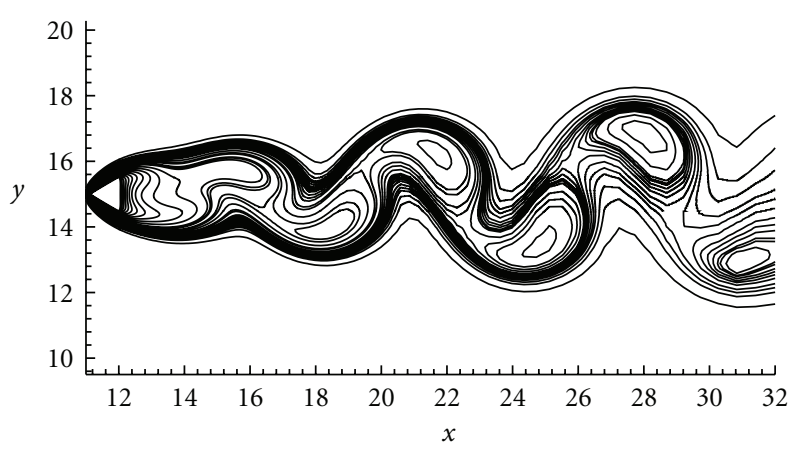

(b)

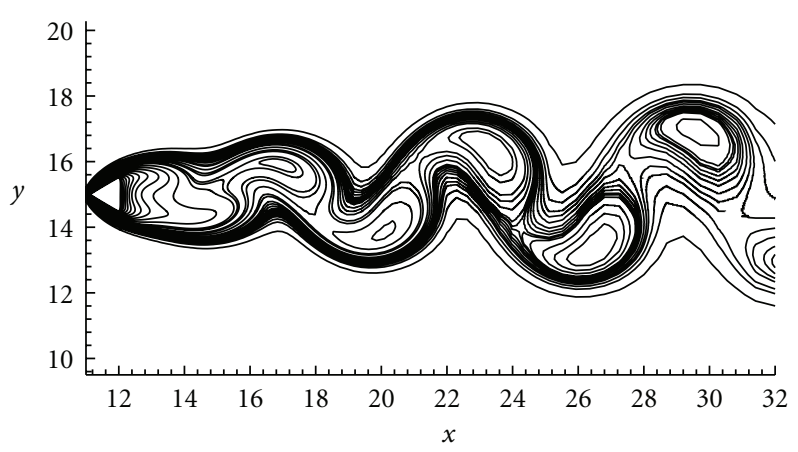

(c)

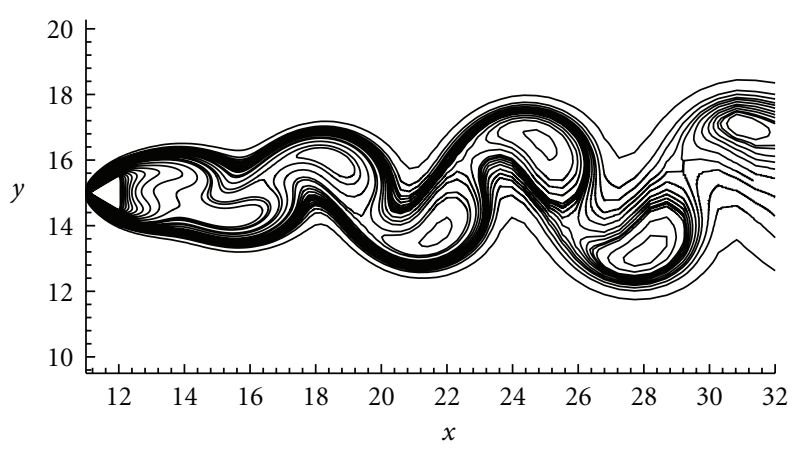

(d)

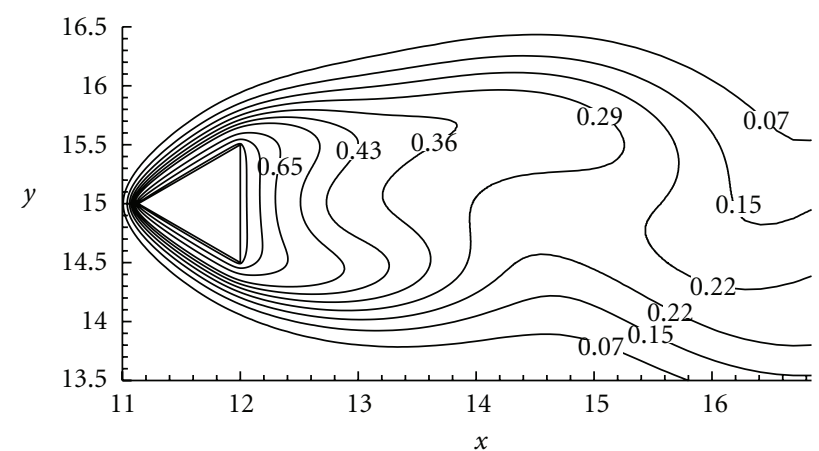

(e)

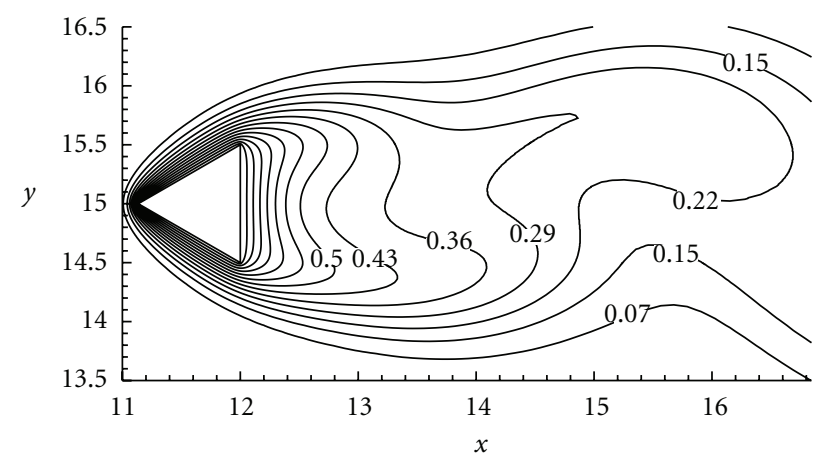

(f)

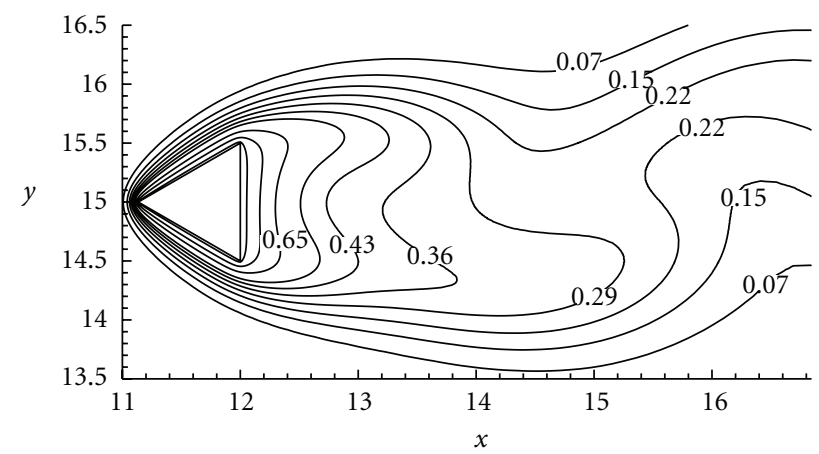

(g)

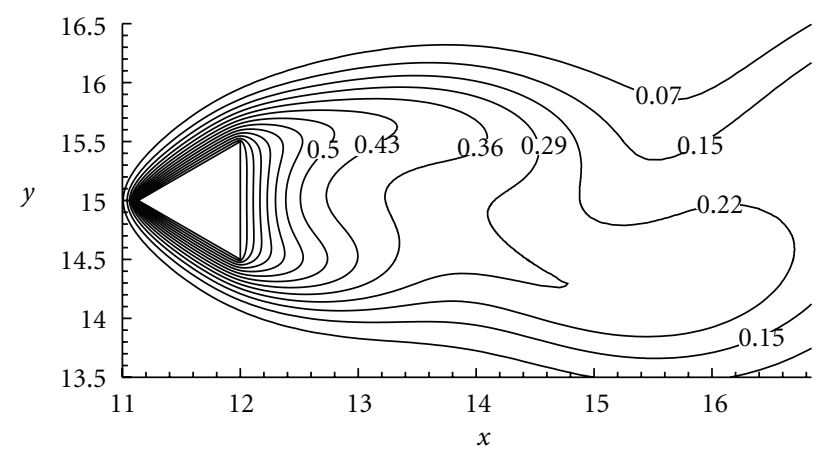

(h)

FIgURE 3: Instantaneous isotherm profiles at the Reynolds number of 50: complete views (a-d) along with the magnified views of isotherms in the vicinity of the triangular cylinder $(\mathrm{e}-\mathrm{h})$. 


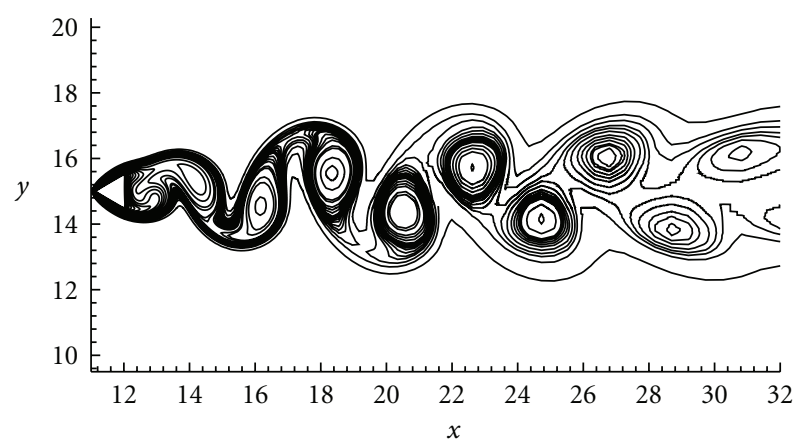

(a)

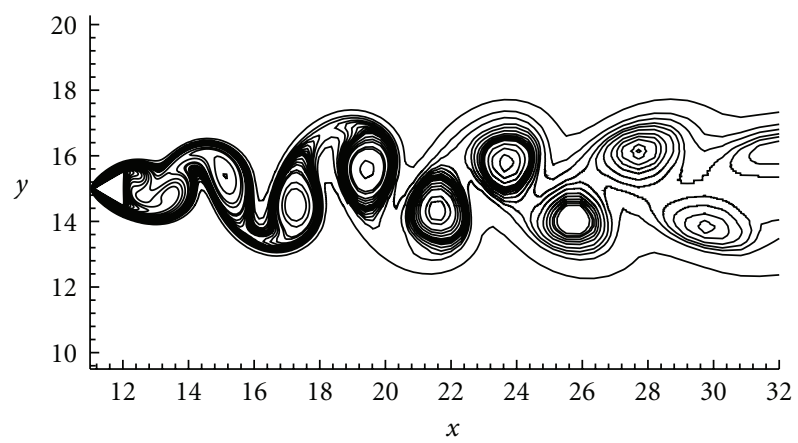

(b)

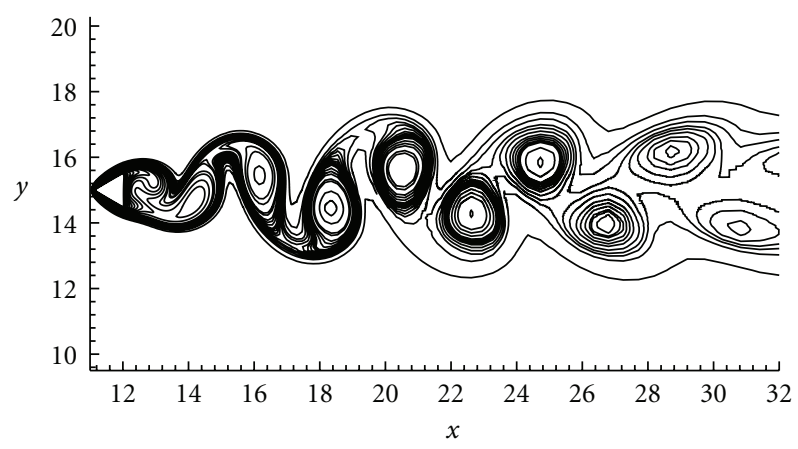

(c)

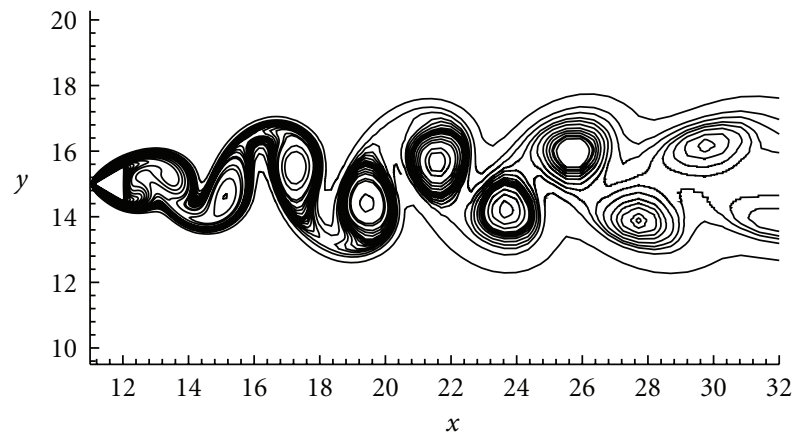

(d)

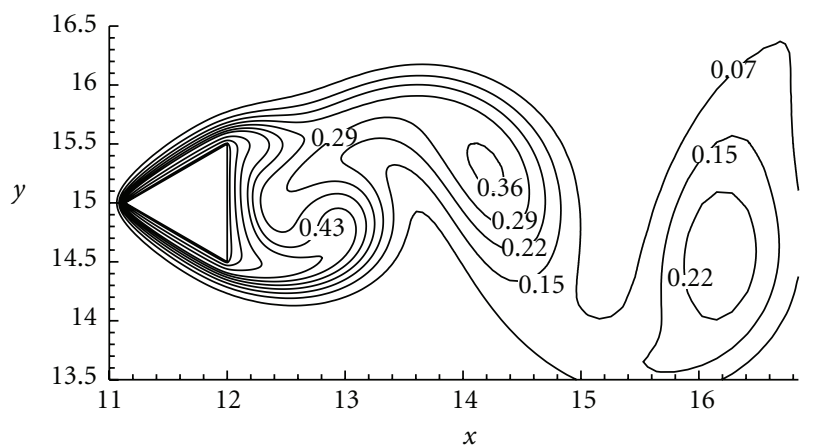

(e)

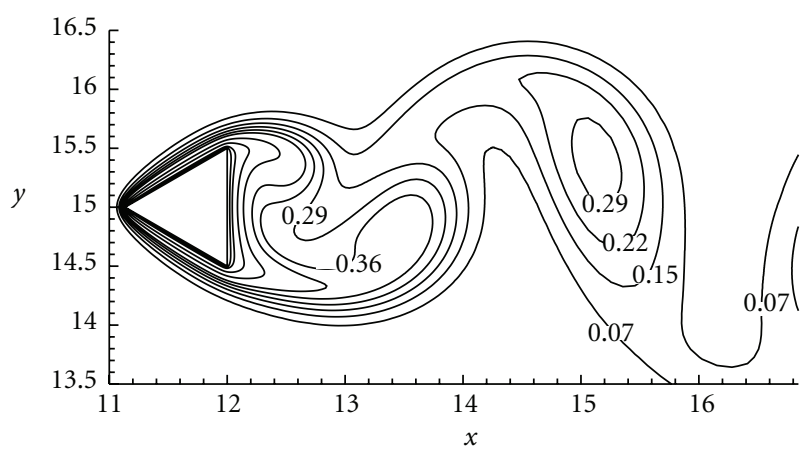

(f)

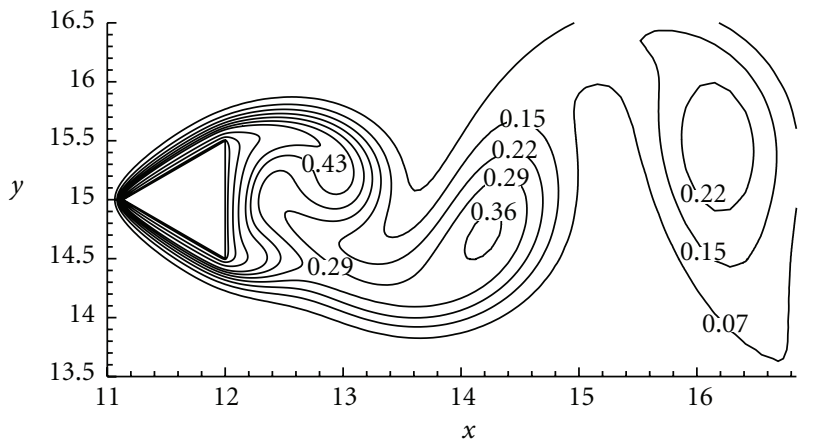

(g)

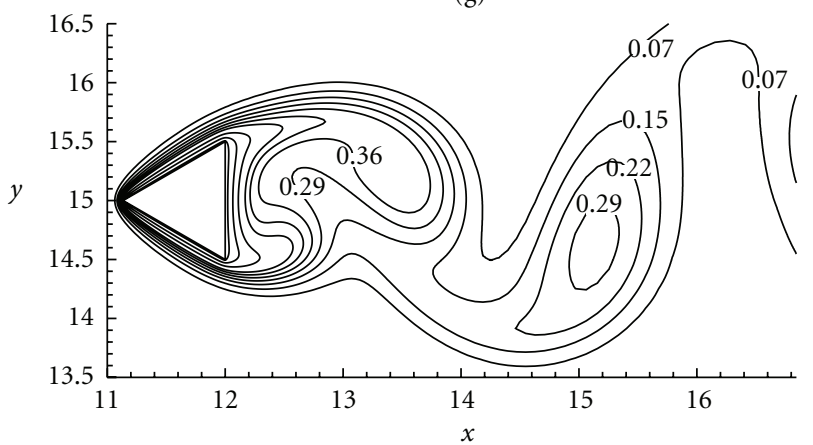

(h)

FIGURE 4: Instantaneous isotherm profiles at the Reynolds number of 100: complete views (a-d) along with the magnified views of isotherms in the vicinity of the triangular cylinder $(\mathrm{e}-\mathrm{h})$. 


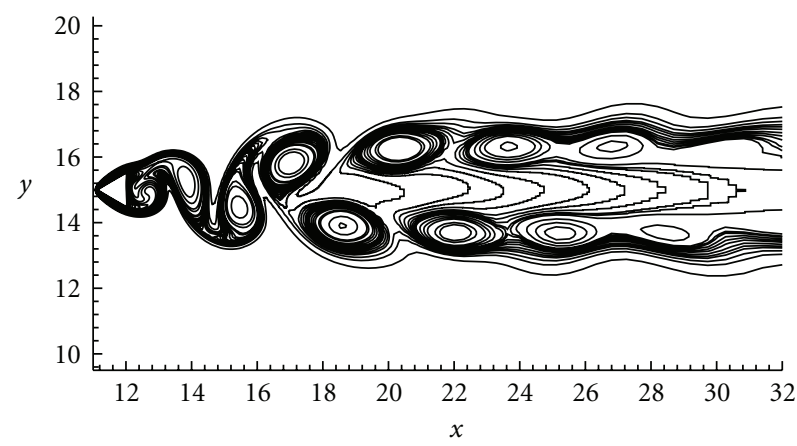

(a)

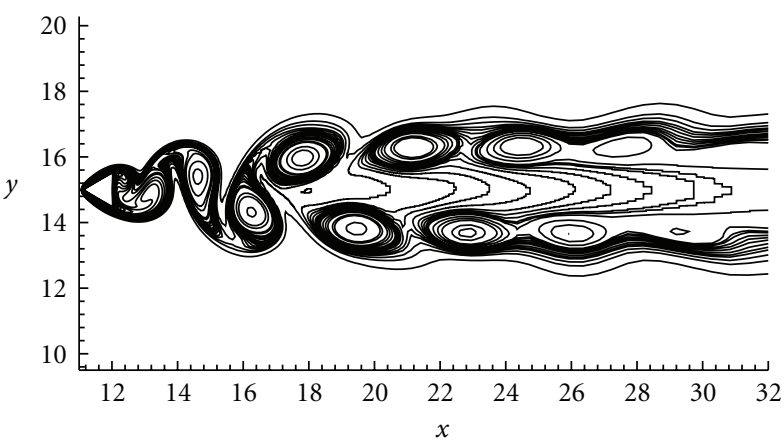

(b)

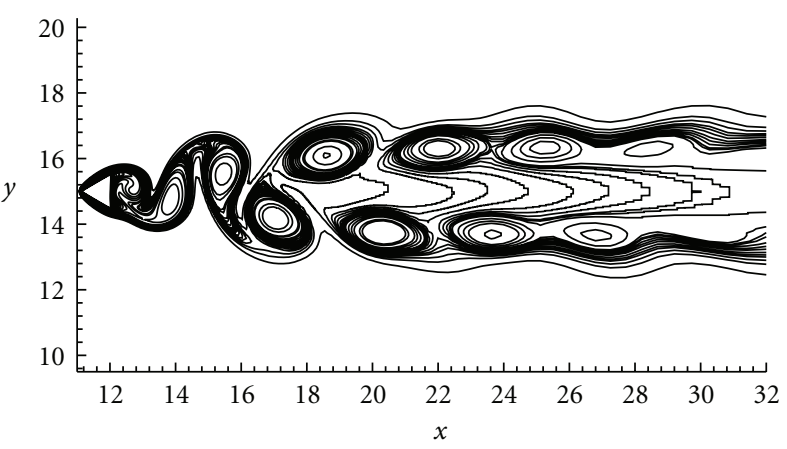

(c)

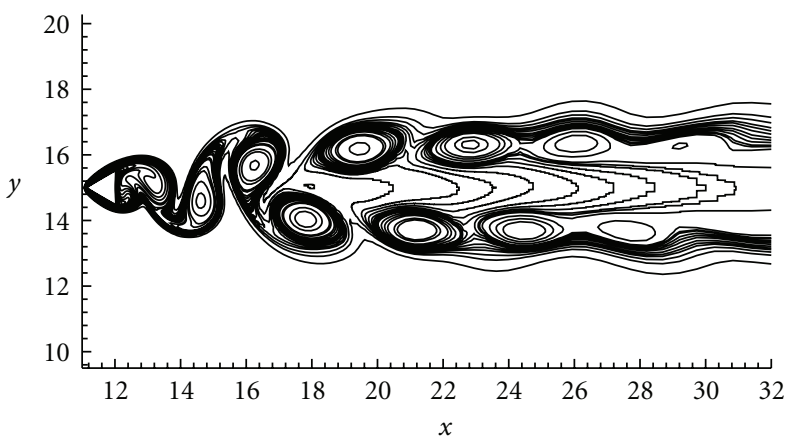

(d)

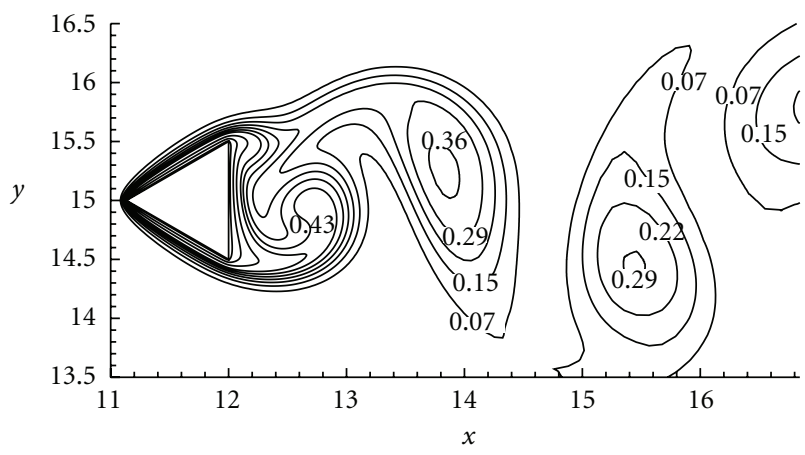

(e)

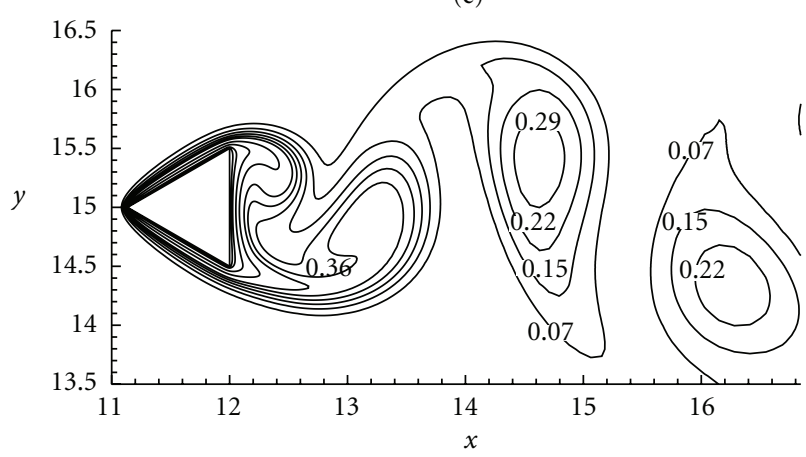

(f)

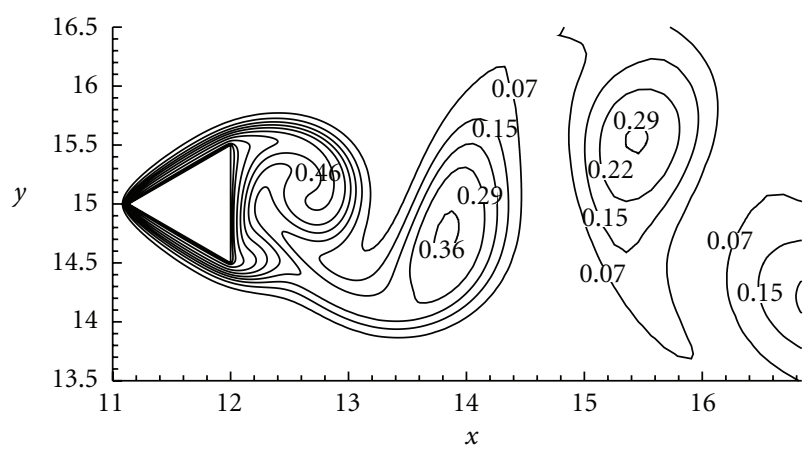

(g)

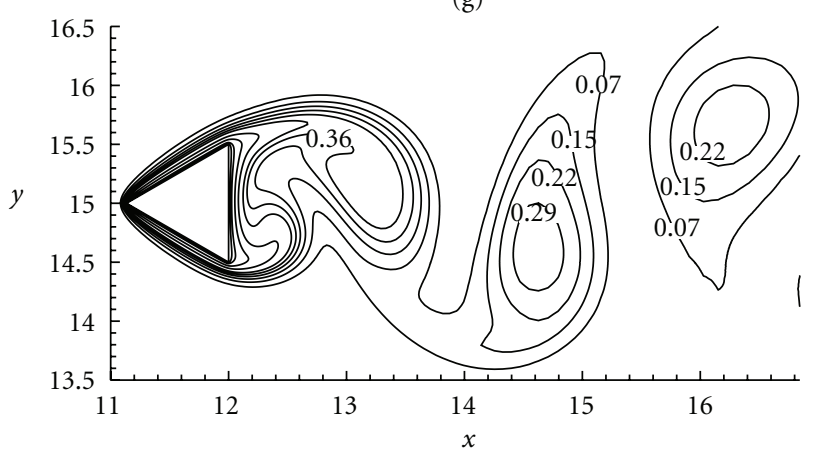

(h)

FIGURE 5: Instantaneous isotherm profiles at the Reynolds number of 150: complete views (a-d) along with the magnified views of isotherms in the vicinity of the triangular cylinder $(\mathrm{e}-\mathrm{h})$. 


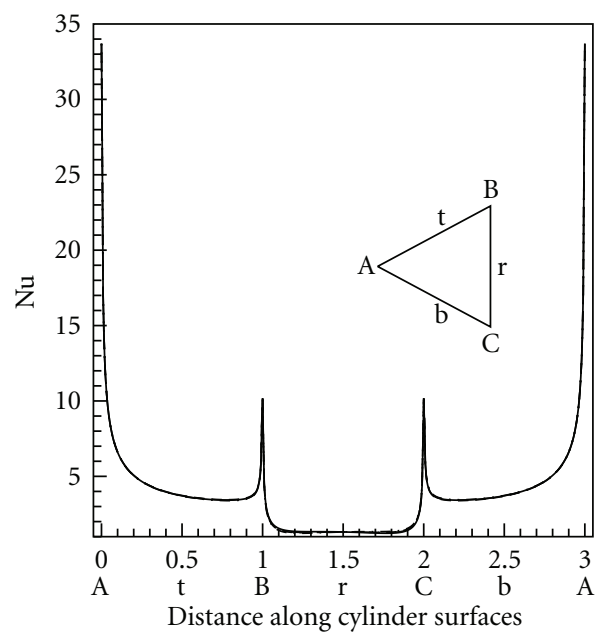

(a) $\mathrm{Re}=50$

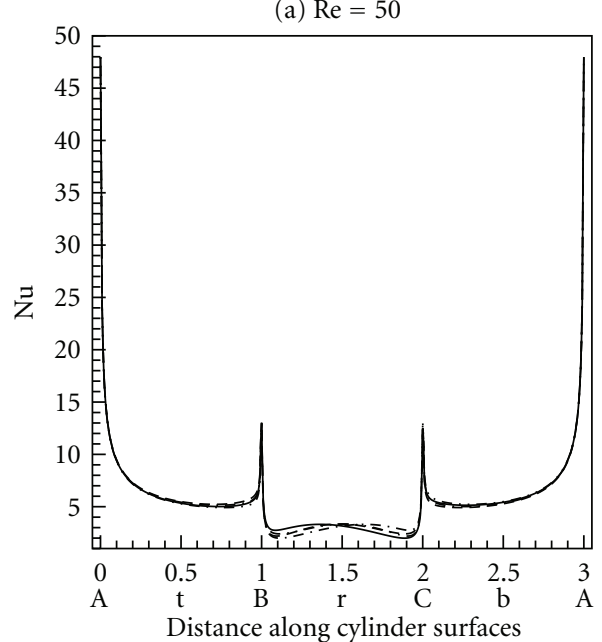

(b) $\operatorname{Re}=100$

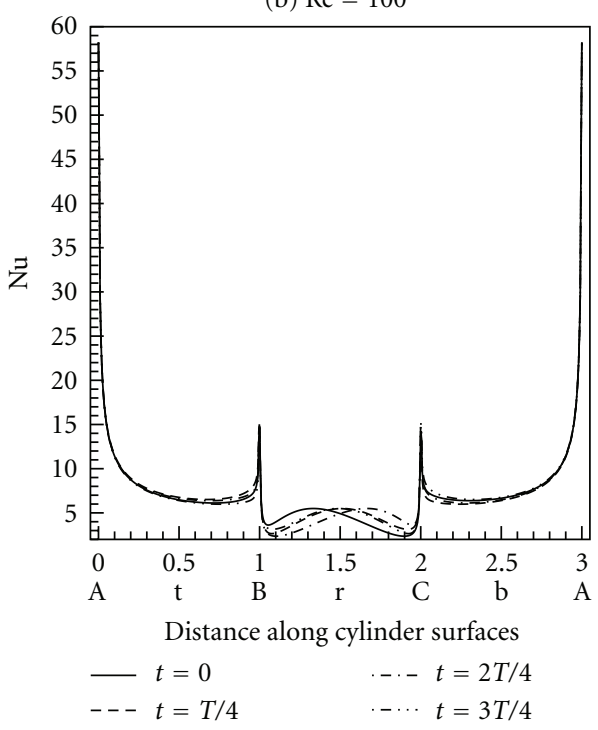

(c) $\operatorname{Re}=150$

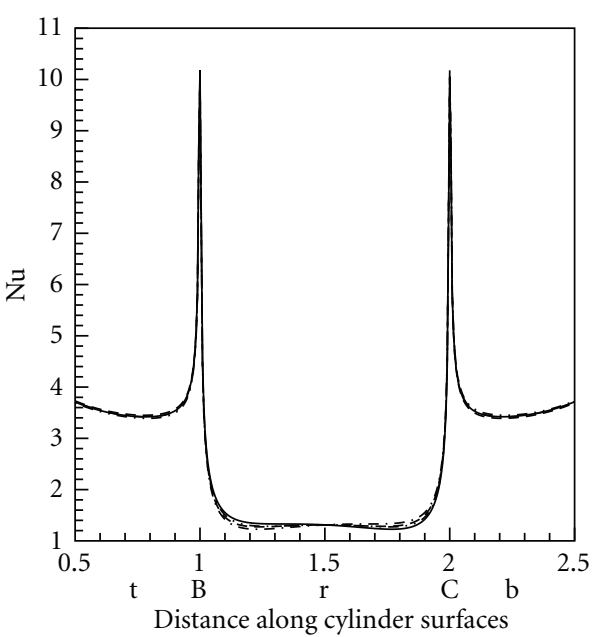

(d) $\operatorname{Re}=50$

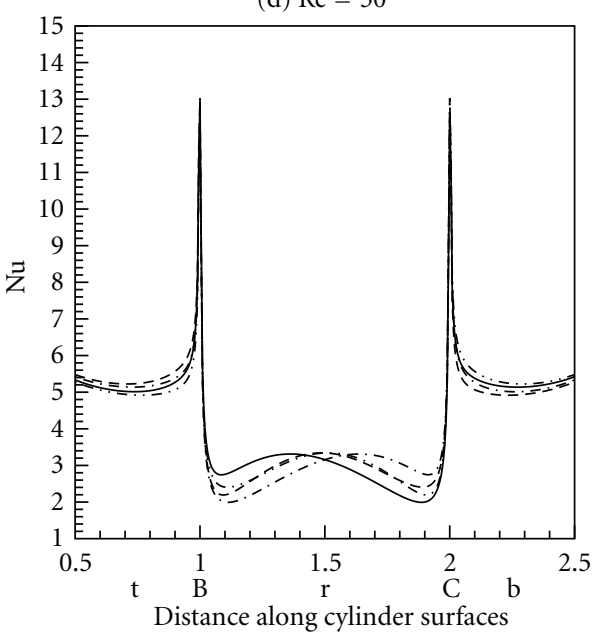

(e) $\operatorname{Re}=100$

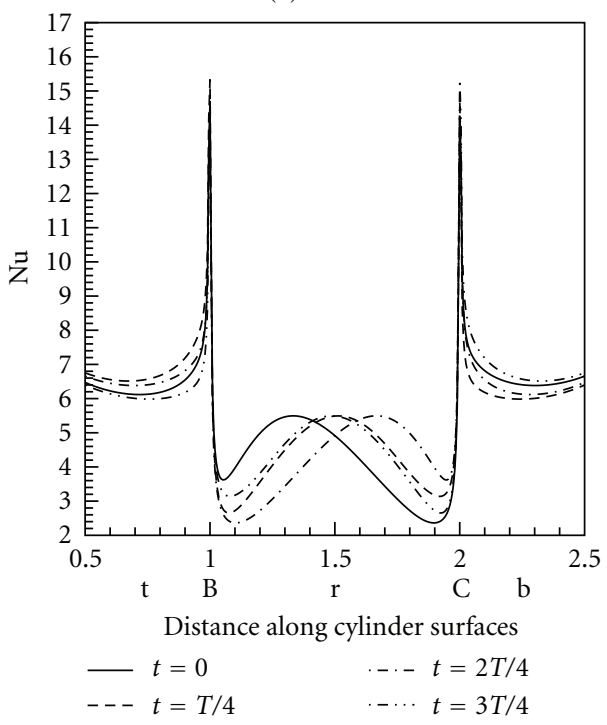

(f) $\operatorname{Re}=150$

FIGURE 6: Instantaneous local Nusselt number around the triangular cylinder surfaces $(\mathrm{a}-\mathrm{c})$ complete and $(\mathrm{d}-\mathrm{f})$ magnified views for Re $=$ 50, 100, and 150 . 


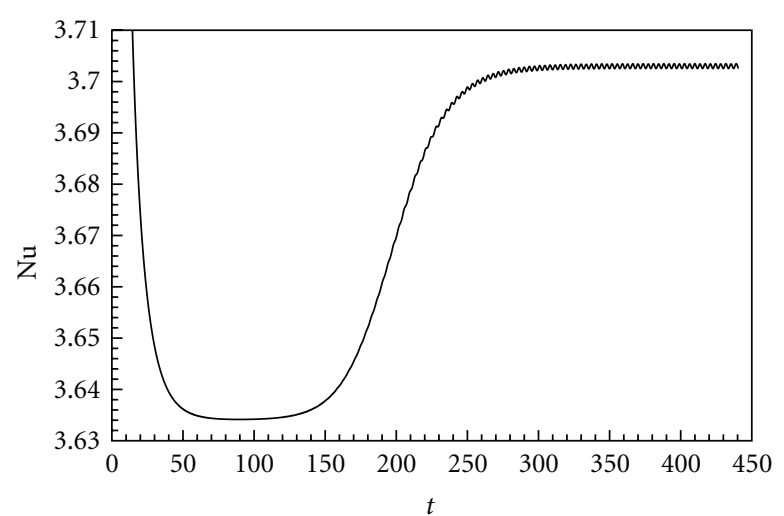

(a) $\mathrm{Re}=50$

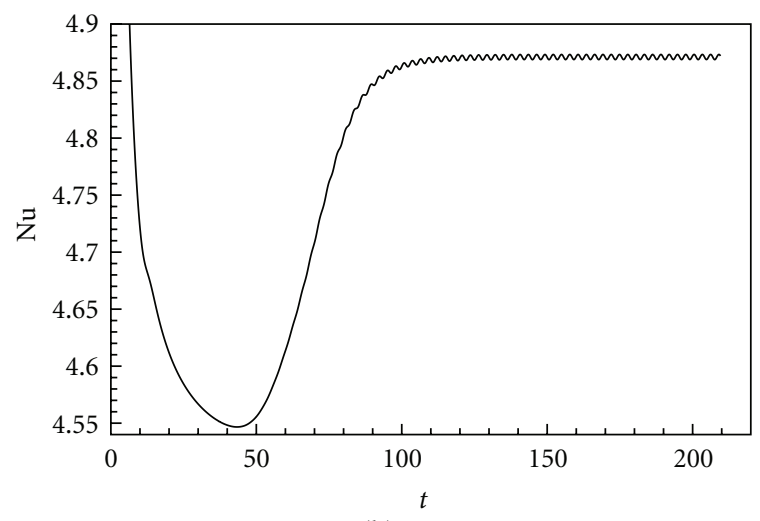

(b) $\operatorname{Re}=80$

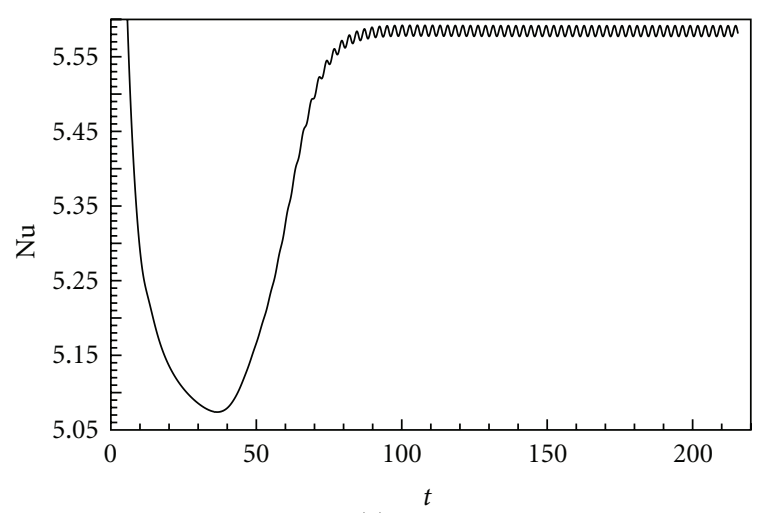

(c) $\operatorname{Re}=100$

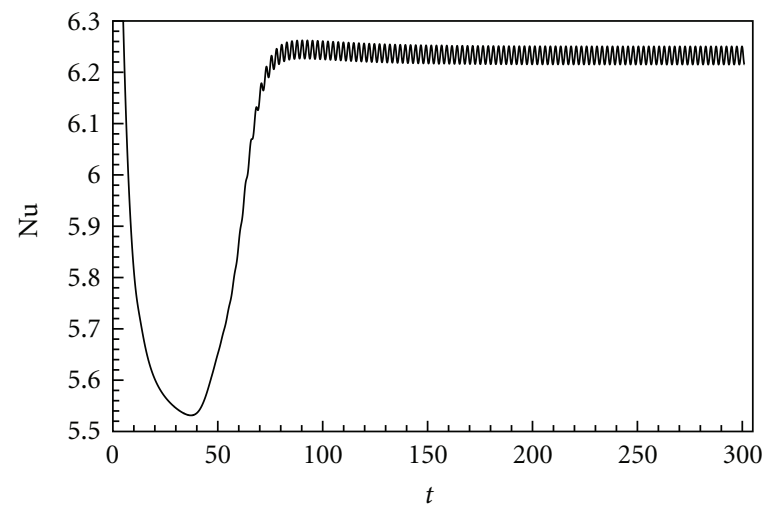

(d) $\operatorname{Re}=120$

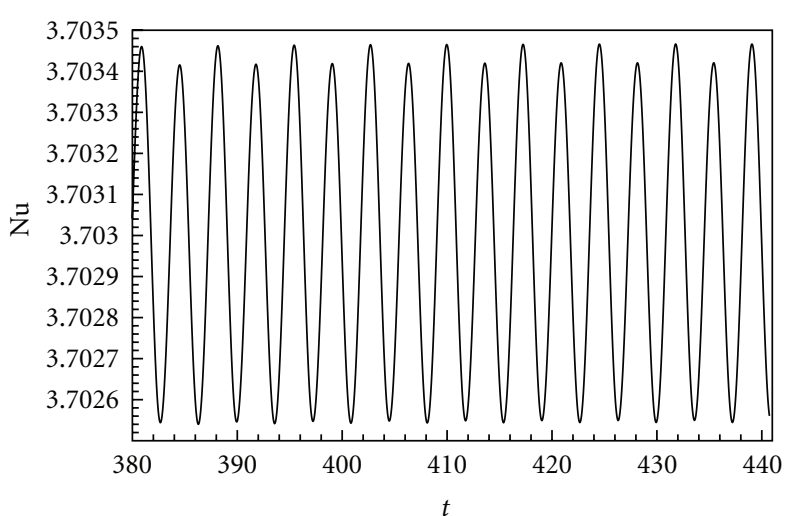

(f) $\operatorname{Re}=50$

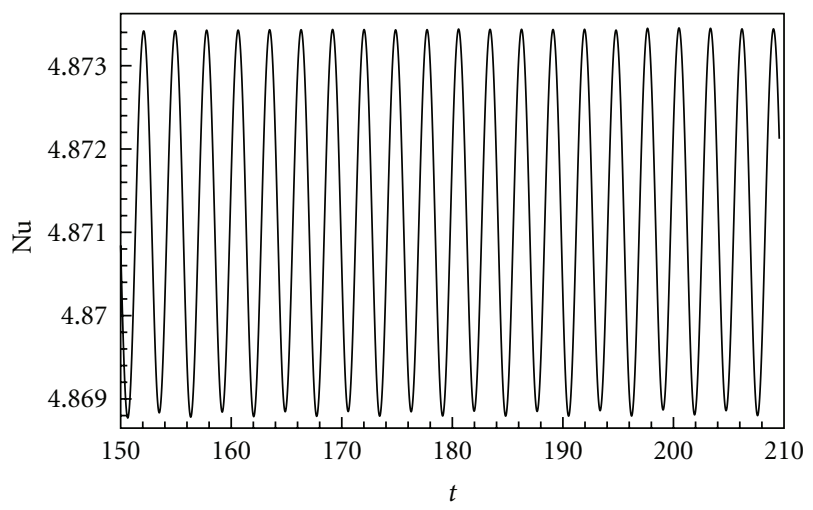

(g) $\operatorname{Re}=80$

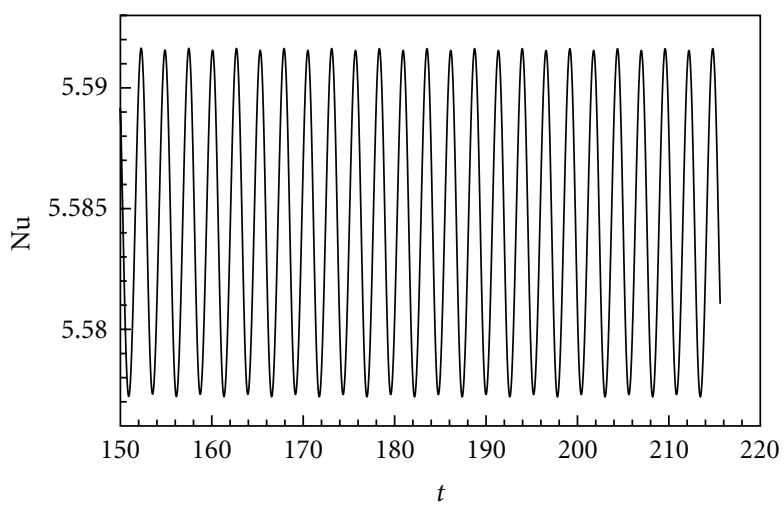

(h) $\mathrm{Re}=100$

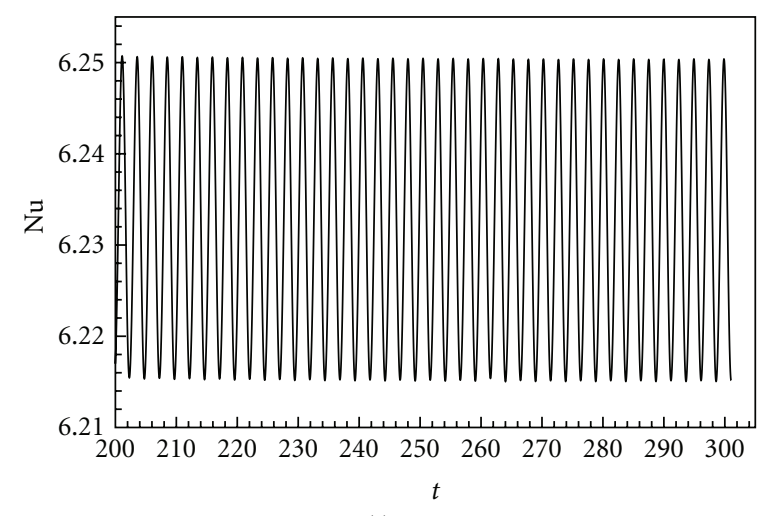

(i) $\operatorname{Re}=120$

Figure 7: Continued. 


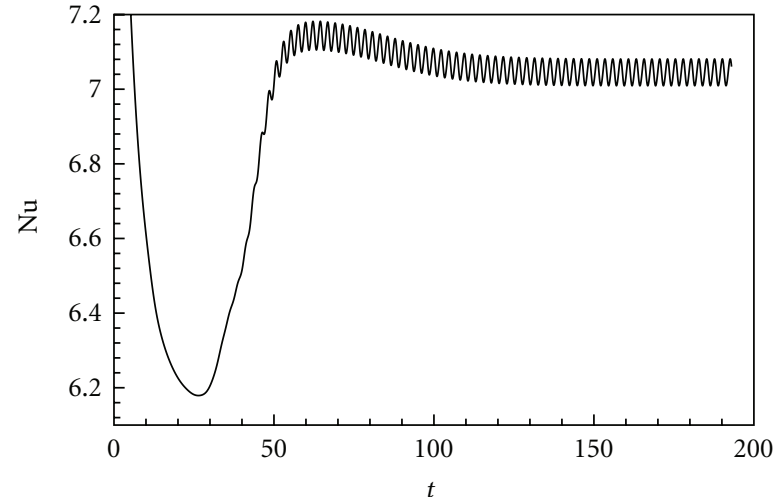

(e) $\operatorname{Re}=150$

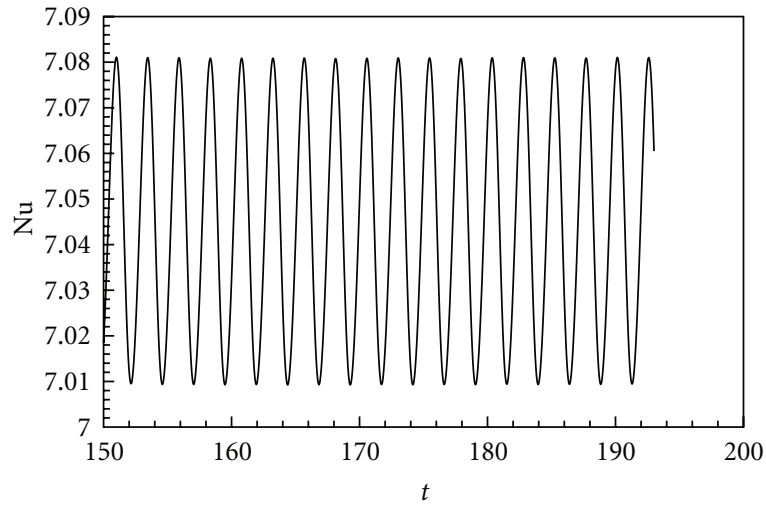

(j) $\operatorname{Re}=150$

Figure 7: Time history of Nusselt number (a-e) complete and (f-j) magnified views for $\operatorname{Re}=50,80,100,120$, and 150 .

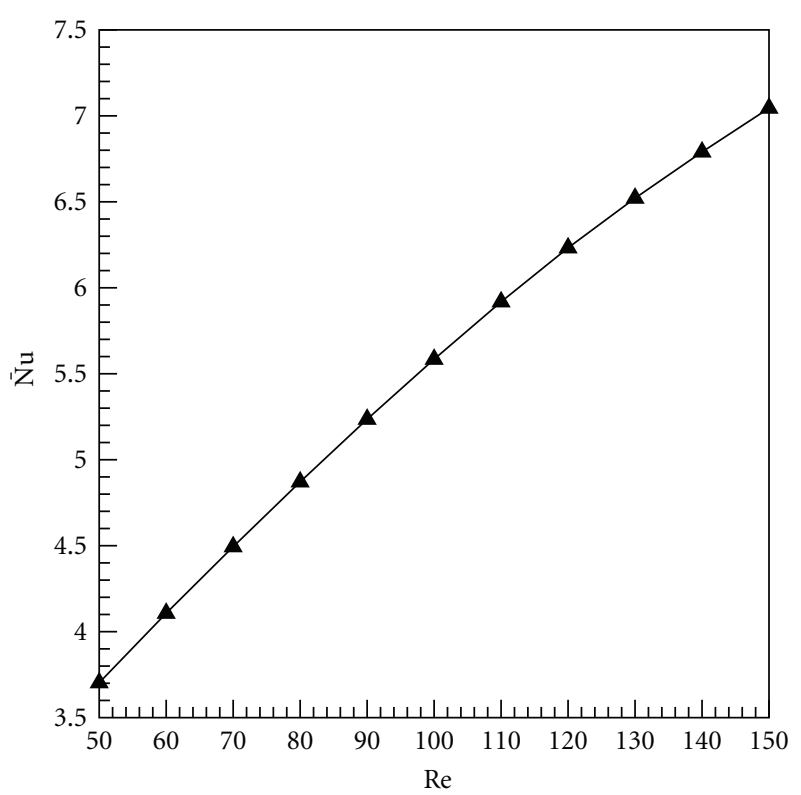

(a)

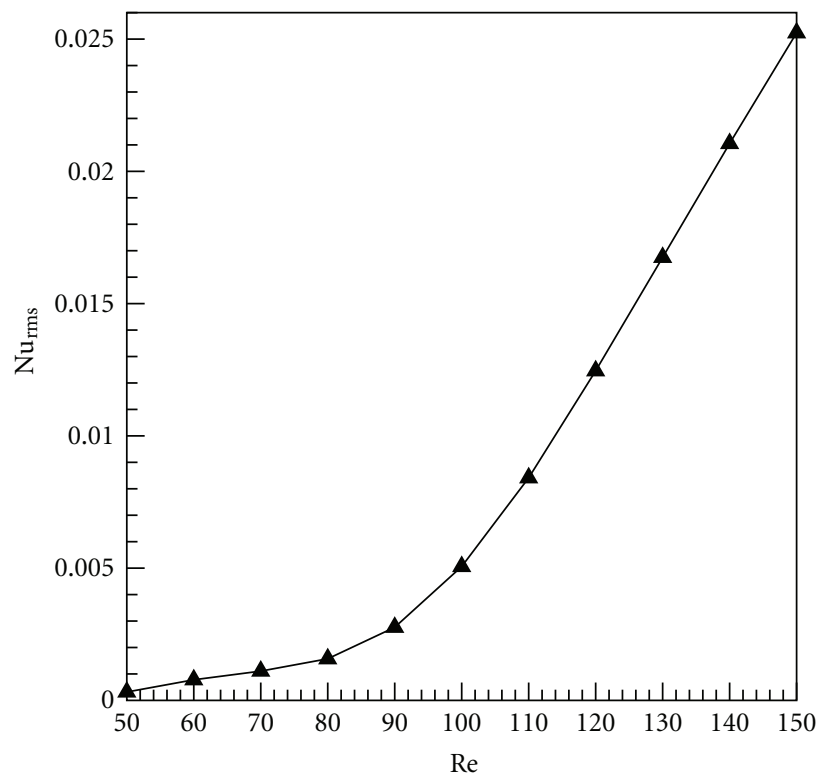

(b)

FIGURE 8: Variation of (a) time-averaged Nusselt number and (b) rms value of Nusselt number with Reynolds number.

Nusselt number on the top surface (i.e., near the top-rear corner) of the triangular cylinder (Figure 6) for all the four successive moments of time considered here at different values of the Reynolds number. Similar to the top surface, the local minimum in the Nusselt number on the bottom surface exists near the bottom-rear corner of the triangular cylinder. On the other hand, a local maximum in the value of the local Nusselt number on the rear surface of the triangular cylinder can also be seen, especially at higher values of Reynolds number in Figure 6 and is increasing with increasing value of the Reynolds number. Also, this maximum in the value of the local Nusselt number on the rear surface of the triangular obstacle fluctuates from the top-rear corner to bottom-rear corner and vice versa for the four successive moments of time, which span over the whole period. This is due the symmetrically shedding of vortices from the top-rear and bottom-rear corners of the triangular obstacle. It is also clear from these figures that the distribution of local Nusselt number on the rear surface affects more than that of the top and bottom surfaces of the triangular obstacle with time due to vortex shedding in the unconfined unsteady regime.

4.4. Time History of Nusselt Number. The temporal variations of the Nusselt number for the values of the Reynolds number of 50, 80, 100, 120, and 150 are shown in Figures $7(\mathrm{a}-\mathrm{e})$. However, the corresponding magnified views of the time history of the Nusselt number at different values of the Reynolds number are presented in Figures $7(\mathrm{f}-\mathrm{j})$. Here, the 


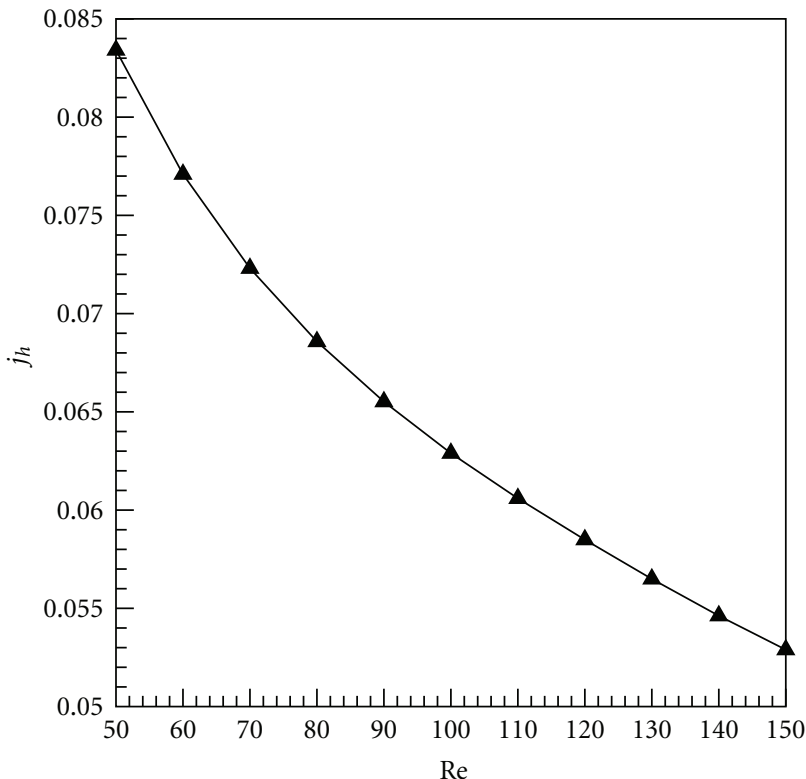

Figure 9: Variation of the Colburn heat transfer factor $\left(j_{h}\right)$ with Reynolds number for the fixed value of the Prandtl number.

instantaneous values of the Nusselt number are calculated at each time step and plotted versus time. Initially, the value of the Nusselt number fluctuates (Figures $7(\mathrm{a}-\mathrm{e})$ ) and finally stabilizes (Figures $7(\mathrm{f}-\mathrm{j})$ ) with time for all the cases considered. It is also clear from these figures that Nusselt number increases with increasing value of the Reynolds number.

4.5. Average Nusselt Number. In this study, the average Nusselt number is calculated by averaging the local Nusselt number over the surfaces of the long equilateral triangular obstacle. The time-averaged Nusselt number is calculated by averaging 10 cycles beyond the time the asymptotic shedding frequency of the Karman vortex is attained. The time-averaged values of the Nusselt number are presented in Figure 8(a). The time-averaged Nusselt number increases monotonically with increasing value of the Reynolds number for the Prandtl number of 0.71. Further, these time-averaged values of the Nusselt number are utilized to obtain the simple heat transfer correlation for the range of physical parameters covered here;

$$
\overline{\mathrm{Nu}}=0.3638 \times \mathrm{Re}^{0.5924} .
$$

The above correlation has the maximum deviation of less than $0.5 \%$ with the present computed results for the range of conditions as $50 \leq \mathrm{Re} \leq 150$ and $\mathrm{Pr}=0.71$.

The rms values of the Nusselt number are also calculated here, as presented in Figure 8(b). The rms value of the Nusselt number increases with increasing value of the Reynolds number. In order to give further insights, the present results are also presented in terms of the usual Colburn heat transfer factor $\left(j_{h}\right)$ to show the functional dependency of the flow and heat transfer parameters [20-22, 35]. Figure 9 shows the variation of the $j_{h}$-factor with Reynolds number. The $j_{h^{-}}$ factor varies approximately linearly with Reynolds number for the fixed value of the Prandtl number for the range of conditions covered here.

\section{Conclusions}

In the present study, the unsteady heat transfer across a long equilateral triangular cylinder is simulated for the range of conditions: Reynolds number $=50-150$ (in the steps of 10) and Prandtl number $=0.71$ (air) in the unbounded unsteady flow regime. The detailed heat transfer characteristics around the triangular obstacle are presented by instantaneous temperature profiles and by the temporal variation of the Nusselt number with Reynolds number. The local Nusselt number at each corner of the triangular cylinder increases with increasing value of the Reynolds number. The time-averaged Nusselt number increases monotonically with increasing value of the Reynolds number for the fixed value of the Prandtl number. Finally, simple heat transfer correlation is obtained for the above range of conditions.

\section{Nomenclature}

$d: \quad$ side of an equilateral triangular cylinder, $\mathrm{m}$

$\bar{C}_{D}$ : time-averaged drag coefficient

$c_{p}$ : $\quad$ specific heat of the fluid, $\mathrm{J} / \mathrm{kg} \mathrm{K}$

$h$ : local convective heat transfer coefficient, $\mathrm{W} / \mathrm{m}^{2} \mathrm{~K}$

$\bar{h}$ : average convective heat transfer coefficient, $\mathrm{W} / \mathrm{m}^{2} \mathrm{~K}$

$j_{h}: \quad$ Colburn factor for heat transfer $\left[=\overline{\mathrm{Nu}} /\left(\operatorname{Re} \times \operatorname{Pr}^{1 / 3}\right)\right]$

$k$ : thermal conductivity of the fluid, $\mathrm{W} / \mathrm{m} \mathrm{K}$

$L_{1}$ : length of the computational domain, $\mathrm{m}$

$L_{2}$ : height of the computational domain, $\mathrm{m}$

$n$ : $\quad$ cylinder surface normal direction

$\mathrm{Nu}$ : local Nusselt number $(=h d / k)$

Nu: $\quad$ time-averaged Nusselt number $(=\bar{h} d / k)$

$\mathrm{Nu}_{\mathrm{rms}}$ : root mean square (rms) value of Nusselt number

$p: \quad$ pressure $\left[=p^{*} /\left(\rho U_{\infty}^{2}\right)\right]$

Pr: $\quad$ Prandtl number $\left[=\mu c_{p} / k\right]$

Re: $\quad$ Reynolds number $\left[=d U_{\infty} \rho / \mu\right]$

St: $\quad$ Strouhal number

$t: \quad$ time $\left[=t^{*} /\left(d / U_{\infty}\right)\right]$

$T: \quad$ time period

$T_{w}^{*}$ : constant wall temperature at the surface of the cylinder, $\mathrm{K}$

$T_{\infty}: \quad$ temperature of the fluid at the inlet, $\mathrm{K}$

$U_{\infty}$ : uniform velocity of the fluid at the inlet, $\mathrm{m} / \mathrm{s}$

$V_{x}: \quad$ component of velocity in the $x$-direction $\left[=V_{x}^{*} / U_{\infty}\right]$

$V_{y}: \quad$ component of velocity in the $y$-direction $\left[=V_{y}^{*} / U_{\infty}\right]$

$x: \quad$ stream-wise coordinate $\left(=x^{*} / d\right)$

$X_{d}$ : downstream distance of the cylinder, $\mathrm{m}$

$X_{u}$ : upstream distance of the cylinder, $\mathrm{m}$

$y: \quad$ transverse coordinate $\left(=y^{*} / d\right)$.

\section{Greek Symbols}

$\theta$ : temperature $\left[=\left(T^{*}-T_{\infty}\right) /\left(T_{w}^{*}-T_{\infty}\right)\right]$

$\mu$ : dynamic viscosity of the fluid, Pa s 
$\rho$ : density of the fluid, $\mathrm{kg} / \mathrm{m}^{3}$

$\delta$ : smallest cell size, $\mathrm{m}$.

\section{Subscripts}

$b$ : bottom surface of the triangular cylinder

$r$ : rear surface of the triangular cylinder

$t$ : top surface of the triangular cylinder

$w$ : surface of the triangular cylinder

$\infty$ : inlet condition.

\section{Superscript}

$*$ : dimensional variable.

\section{References}

[1] R. P. Chhabra, "Hydrodynamics of non-spherical particles in non-Newtonian fluids," in Handbook of Applied Polymer Processing Technology, N. P. Cheremisinoff, Ed., chapter 1, Marcel Dekker, 1996.

[2] S. Goujon-Durand, P. Jenffer, and J. E. Wesfreid, "Downstream evolution of the Bénard-von Kármán instability," Physical Review E, vol. 50, no. 1, pp. 308-313, 1994.

[3] T. S. Lee, "Early stages of an impulsively started unsteady laminar flow past tapered trapezoidal cylinders," International Journal for Numerical Methods in Fluids, vol. 26, no. 10, pp. 1181-1203, 1998.

[4] Y. J. Chung and S. H. Kang, "Laminar vortex shedding from a trapezoidal cylinder with different height ratios," Physics of Fluids, vol. 12, no. 5, pp. 1251-1254, 2000.

[5] R. Kahawita and P. Wang, "Numerical simulation of the wake flow behind trapezoid bluff bodies," Computers and Fluids, vol. 31, no. 1, pp. 99-112, 2002.

[6] C. P. Jackson, "A finite-element study of the onset of vortex shedding in flow past variously shaped bodies," The Journal of Fluid Mechanics, vol. 182, pp. 23-45, 1987.

[7] B. J. A. Zielinska and J. E. Wesfreid, "On the spatial structure of global modes in wake flow," Physics of Fluids, vol. 7, no. 6, pp. 1418-1424, 1995.

[8] J. E. Wesfreid, S. Goujon-Durand, and B. J. A. Zielinska, "Global mode behavior of the streamwise velocity in wakes," Journal de Physique II, vol. 6, no. 10, pp. 1343-1357, 1996.

[9] A. K. De and A. Dalal, "Numerical simulation of unconfined flow past a triangular cylinder," International Journal for Numerical Methods in Fluids, vol. 52, no. 7, pp. 801-821, 2006.

[10] A. Dalal, V. Eswaran, and G. Biswas, "A finite-volume method for Navier-Stokes equations on unstructured meshes," Numerical Heat Transfer B, vol. 54, no. 3, pp. 238-259, 2008.

[11] H. Chattopadhyay, "Augmentation of heat transfer in a channel using a triangular prism," International Journal of Thermal Sciences, vol. 46, no. 5, pp. 501-505, 2007.

[12] H. Abbassi, S. Turki, and S. Ben Nasrallah, "Numerical investigation of forced convection in a plane channel with a built-in triangular prism," International Journal of Thermal Sciences, vol. 40, no. 7, pp. 649-658, 2001.

[13] A. K. De and A. Dalal, "Numerical study of laminar forced convection fluid flow and heat transfer from a triangular cylinder placed in a channel," Journal of Heat Transfer, vol. 129, no. 5, pp. 646-656, 2007.

[14] R. W. David and E. F. Moore, "A numerical study of vortex shedding from rectangles," Journal of Fluid Mechanics, vol. 116, pp. 475-506, 1982.
[15] T. Igarashi, "Fluid flow and heat transfer around rectangular cylinders (the case of a width/height ratio of a section of 0.33 1.5)," International Journal of Heat and Mass Transfer, vol. 30, no. 5, pp. 893-901, 1987.

[16] A. Okajima, "Numerical simulation of flow around rectangular cylinders," Journal of Wind Engineering and Industrial Aerodynamics, vol. 33, no. 1-2, pp. 171-180, 1990.

[17] S. Nitin and R. P. Chhabra, "Non-isothermal flow of a power law fluid past a rectangular obstacle (of aspect ratio $1 \times 2$ ) in a channel: drag and heat transfer," International Journal of Engineering Science, vol. 43, no. 8-9, pp. 707-720, 2005.

[18] B. Paliwal, A. Sharma, R. P. Chhabra, and V. Eswaran, "Power law fluid flow past a square cylinder: momentum and heat transfer characteristics," Chemical Engineering Science, vol. 58, no. 23-24, pp. 5315-5329, 2003.

[19] A. Sharma and V. Eswaran, "Heat and fluid flow across a square cylinder in the two-dimensional laminar flow regime," Numerical Heat Transfer A, vol. 45, no. 3, pp. 247-269, 2004.

[20] A. K. Dhiman, R. P. Chhabra, A. Sharma, and V. Eswaran, "Effects of Reynolds and prandtl numbers on heat transfer across a square cylinder in the steady flow regime," Numerical Heat Transfer A, vol. 49, no. 7, pp. 717-731, 2006.

[21] A. K. Dhiman, R. P. Chhabra, and V. Eswaran, "Heat transfer to power-law fluids from a heated square cylinder," Numerical Heat Transfer A, vol. 52, no. 2, pp. 185-201, 2007.

[22] A. K. Sahu, R. P. Chhabra, and V. Eswaran, "Effects of reynolds and prandtl numbers on heat transfer from a square cylinder in the unsteady flow regime," International Journal of Heat and Mass Transfer, vol. 52, no. 3-4, pp. 839-850, 2009.

[23] V. T. Morgan, "The overall convective heat transfer from smooth circular cylinders," Advances in Heat Transfer, vol. 11, pp. 199-264, 1975.

[24] R. A. Ahmad, "Steady-state numerical solution of the navierstokes and energy equations around a horizontal cylinder at moderate reynolds numbers from 100 to 500," Heat Transfer Engineering, vol. 17, no. 1, pp. 31-81, 1996.

[25] E. R. G. Eckert and E. Soehngen, "Distribution of heat transfer coefficient around circular cylinder in cross flow at Reynolds numbers from 20 to 500," ASME Transactions, vol. 74, pp. 343$347,1952$.

[26] K. M. Krall and E. R. G. Eckert, "Local heat transfer around a cylinder at low Reynolds number," Journal of Heat Transfer, vol. 95, no. 2, pp. 273-275, 1973.

[27] T. S. Sarma and S. P. Sukhatme, "Local heat transfer from a horizontal cylinder to air in cross flow: influence of free convection and free stream turbulence," International Journal of Heat and Mass Transfer, vol. 20, no. 1, pp. 51-56, 1977.

[28] G. E. Karniadakis, "Numerical simulation of forced convection heat transfer from a cylinder in crossflow," International Journal of Heat and Mass Transfer, vol. 31, no. 1, pp. 107-118, 1988.

[29] C. F. Lange, F. Durst, and M. Breuer, "Momentum and heat transfer from cylinders in laminar crossflow at $10^{-4} \leq \mathrm{Re} \leq$ 200," International Journal of Heat and Mass Transfer, vol. 41, no. 22, pp. 3409-3430, 1998.

[30] L. Baranyi, "Computation of unsteady momentum and heat transfer from a fixed circular cylinder in laminar flow," The Journal of Computational and Applied Mechanics, vol. 4, pp. 13-25, 2003.

[31] H. Nakamura and T. Igarashi, "Variation of Nusselt number with flow regimes behind a circular cylinder for Reynolds numbers from 70 to 30000," International Journal of Heat and Mass Transfer, vol. 47, no. 23, pp. 5169-5173, 2004. 
[32] J. M. Shi, D. Gerlach, M. Breuer, G. Biswas, and F. Durst, "Heating effect on steady and unsteady horizontal laminar flow of air past a circular cylinder," Physics of Fluids, vol. 16, no. 12, pp. 4331-4345, 2004.

[33] A. A. Soares, J. M. Ferreira, and R. P. Chhabra, "Flow and forced convection heat transfer in crossflow of nonNewtonian fluids over a circular cylinder," Industrial and Engineering Chemistry Research, vol. 44, no. 15, pp. 5815-5827, 2005.

[34] S. A. Isaev, A. I. Leontiev, N. A. Kudryavtsev, T. A. Baranova, and D. A. Lysenko, "Numerical simulation of unsteady-state heat transfer under conditions of laminar transverse flow past a circular cylinder," High Temperature, vol. 43, no. 5, pp. 746759, 2005.

[35] R. P. Bharti, R. P. Chhabra, and V. Eswaran, "Steady forced convection heat transfer from a heated circular cylinder to power-law fluids," International Journal of Heat and Mass Transfer, vol. 50, no. 5-6, pp. 977-990, 2007. 

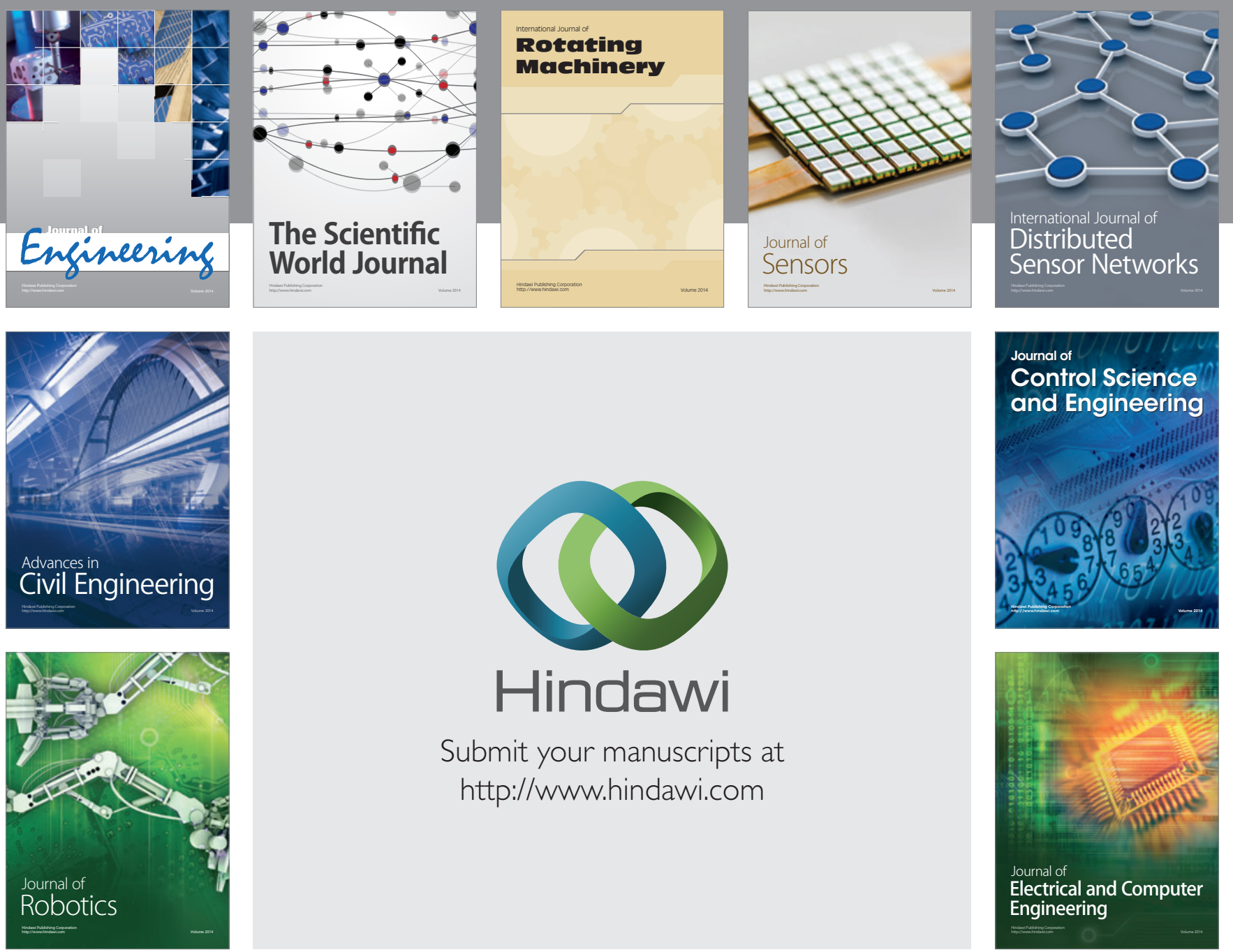

Submit your manuscripts at

http://www.hindawi.com
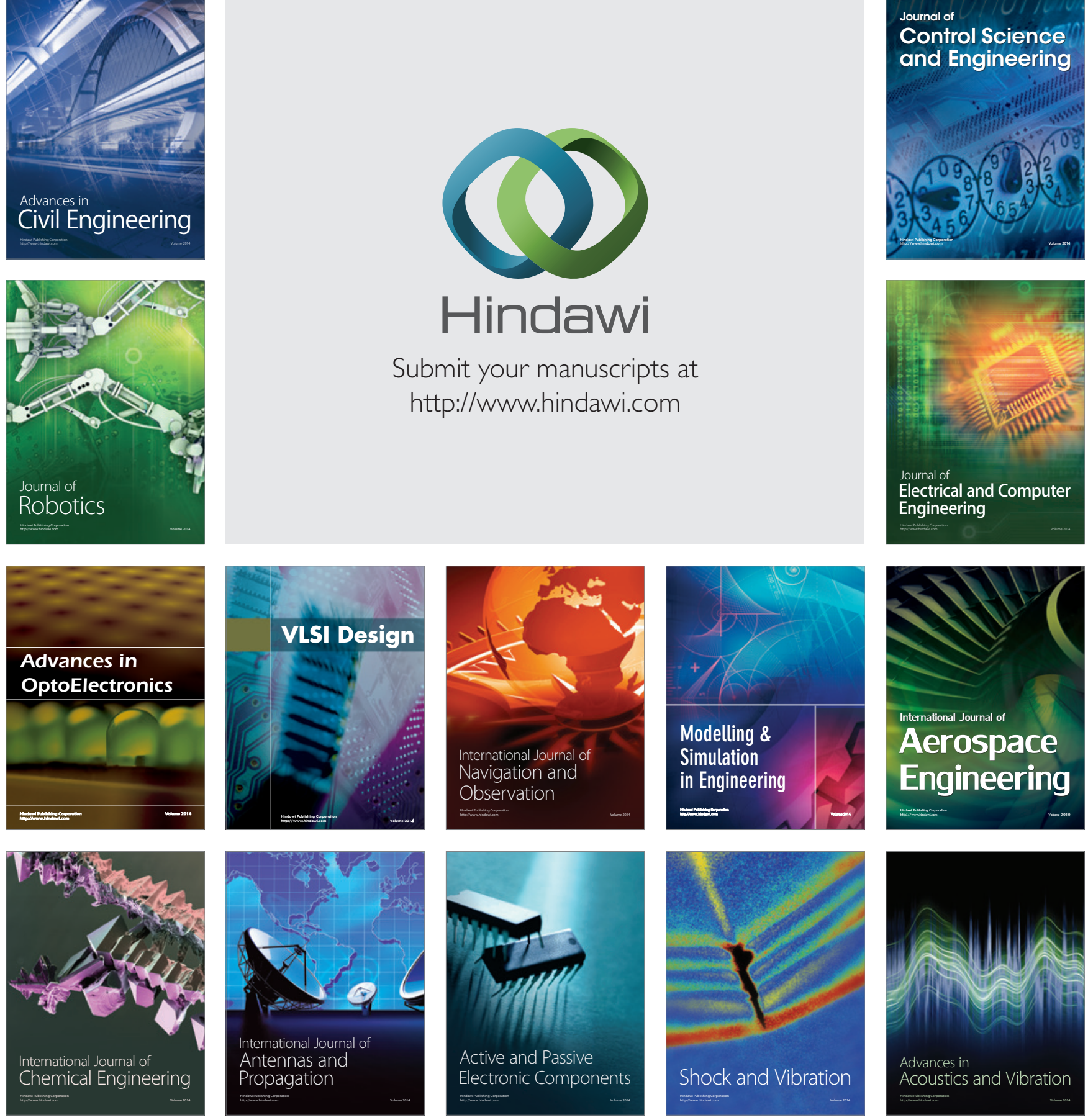